\title{
Special issue on computational intelligence for social mining
}

\author{
David Camacho $^{1} \cdot$ Gema Bello-Orgaz $^{1}$
}

Published online: 21 September 2019

c) Springer-Verlag GmbH Germany, part of Springer Nature 2019

\section{Introduction}

Social media data are generated from a wide number of Internet applications and Web sites, being some of the most popular Facebook, Twitter, LinkedIn, YouTube, Instagram, Google, Tumblr, Flickr, and WordPress. The concept of Social Mining can be defined as those processes and methods that are designed to provide sensitive and relevant knowledge to any user, or company, from social media data sources. These data sources can be characterized by their different formats and contents, their large size, and the online or streamed generation of information. The problem of managing and extracting valuable knowledge from all these social data sources is currently one of the most popular topics in the computer science research, creating new technical and application challenges.

To overcome these challenges, it will be necessary to combine methods and techniques from areas such as data mining (Carneiro et al. 2017), machine learning (Martín et al. 2018), graph mining (Bello-Orgaz et al. 2017), natural language processing (Liao et al. 2018), semantic web (Barhamgi et al. 2018), and big data computing (Bello-Orgaz et al. 2016), among others. On the other hand, Computational intelligence (CI) is a set of nature-inspired computational approaches such as evolutionary computation (BelloOrgaz et al. 2018), swarm intelligence (Gonzalez-Pardo et al. 2017), artificial neural networks (Martín et al. 2017) or fuzzy systems (Villar et al. 2017), which solve complex real-world problem in changing environments. Therefore, CI provides solutions for social mining challenges, allowing the design of algorithmic models to handle complex problems using adaptive mechanisms.

David Camacho

david.camacho@upm.es

Gema Bello-Orgaz

gema.borgaz@upm.es

1 Universidad Politécnica de Madrid, Departamento de Sistemas Informáticos, Calle Alan Turing s/n, 28031 Madrid, Spain
The goal of this special issue is to gather the recent application of computational intelligence approaches/models for social mining. A special emphasis is done to the presentation of novel algorithms, systems, and new real-life applications, as well as survey papers that review the novel technologies and new trends in this area.

\section{Contributions of this issue}

The paper (Sani et al. 2019) presents a novel multiobjective optimization algorithm based on ant colony algorithm to solve the community detection problem in complex networks. In this proposed method, a Pareto archive is considered to store non-dominated solutions found during the algorithm's process maximizing both goals of community fitness and community score in a trade-off manner.

Based on fuzzy possibilistic clustering, in the paper (Abidi et al. 2019) is introduced a new microaggregation method called HM-PFSOM. This proposed method operates through an hybrid manner. That is, the anonymization process is applied per block of similar data. This fact can help to decrease the information loss during the anonymization process. The authors propose to study the distribution of confidential attributes within each sub-dataset. Then, according to the latter distribution, the privacy parameter $\mathrm{k}$ is determined, in such a way to preserve the diversity of confidential attributes within the anonymized microdata. This allows to decrease the disclosure risk of confidential information.

The authors in paper (Valdivia et al. 2019) present a methodology that combines a deep learning algorithm for extracting aspects, a method for clustering related aspects and a subgroup discovery method for obtaining descriptive rules that summarise the sentiment information of set of reviews. Concretely, this methodology aims at depicting negative opinions from three cultural monuments in order to detect those features that need to be improved.

The paper (Xylogiannopoulos et al. 2019) proposes a novel methodology that can detect all frequent and 
nonfrequent patterns in order to remedy the serious problem related to users selecting very weak passwords that compromise their accounts, taking advantage of the availability of real password datasets.

The work presented by authors in (Bui et al. 2019) introduces a novel approach for network management based on data analytics. The main research focuses on how the network configuration can be automatically and adaptively decided, given various dynamic contexts (e.g., network interference, heterogeneity and so on). Specifically, the authors design a context-based data-driven framework for network operation in connected environment which includes three layer architecture: (1) network entity layer; (2) complex semantic analytics layer and (3) action provisioning layer.

Paper (Derbas et al. 2019) presents a tool called Safapp that crawls and analyses data from social media (specially Twitter and blogs) in the context of radicalization detection. This paper are focused on the description of the semantic module of Safapp which is dedicated to the analysis of textual content of social network and blogs, and more specifically on a newly developed module: the event extractor. With this module the authors expect to go one step further in the development of useful tools to track and analyze online propaganda.

In paper (Singh et al. 2019) is presented an overview of sentiment analysis techniques based on recent research, and subsequently explores machine learning (SVM, Navies Bayes, Linear Regression and Random Forest) and feature extraction techniques (POS, BOW and HASS tagging). Further twitter data-sets are scrutinized and pre-processed with proposed framework, which yield intersecting facts about the capabilities and deficiency of sentiment analysis methods.

The authors in paper (Mezni et al. 2019) propose a context-aware Web service recommendation approach with a specific focus of time dimension. First, K-means clustering method is hybridized with a multi-population variant of the wellknown PSO (Particle Swarm Optimization) in order to exclude the less similar users which share few common Web services with the active user in specific contexts. Slope One method is, then, applied to predict the missing ratings in the current context of user. Finally, a recommendation algorithm is proposed in order to return the top-rated services.

Finally, the paper (Chouchani and Abed 2019) shows that information about social influence processes can be used to improve Sentiment Analysis. In particular, the authors use heterogeneous graphs to infer sentiment polarities at userlevel. The results obtained in the work reveal that incorporating such information can indeed lead to statistically significant sentiment classification improvements.

Acknowledgements This special issue has been achieved by a number of fruitful collaborations. We would like to thank the Editor in Chief of the Journal of Ambient Intelligence and Humanized Computing,
Prof. Vincenzo Loia, for his kind support and help during the entire process of publication. In this special issue we processing so many papers in a timely manner would have been impossible but for the timely and active cooperation of the reviewers. The Guest Editors take this opportunity to thank them all. We are most grateful to the authors for their valuable contributions and for their willingness and efforts to improve their papers in accordance with the suggestions and comments from reviewers and editors. Finally, we would like to acknowledge the financial support received from the following research projects: DeepBio (TIN2017-85727-C4-3-P by Spanish Ministry of Economy and Competitiveness), CYNAMON (P2018/TCS-4566 by Comunidad de Madrid), both under the European Regional Development Fund FEDER, and YoungRes project under Grant 823701-ISFP-2017-AGRAD (ISFP-2017-AG-RAD-Radicalisation).

\section{References}

Abidi B, Yahia SB, Perera C (2019) Hybrid microaggregation for privacy preserving data mining. J Ambient Intell Hum Comput. https ://doi.org/10.1007/s12652-018-1122-7

Barhamgi M, Masmoudi A, Lara-Cabrera R, Camacho D (2018) Social networks data analysis with semantics: application to the radicalization problem. J Ambient Intell Hum Comput. https://doi. org/10.1007/s12652-018-0968-z

Bello-Orgaz G, Jung JJ, Camacho D (2016) Social big data: recent achievements and new challenges. Inf Fus 28:45-59. https://doi. org/10.1016/j.inffus.2015.08.005

Bello-Orgaz G, Hernandez-Castro J, Camacho D (2017) Detecting discussion communities on vaccination in Twitter. Future Gen Comput Syst 66:125-136. https://doi.org/10.1016/j.future.2016.06.032

Bello-Orgaz G, Salcedo-Sanz S, Camacho D (2018) A multi-objective genetic algorithm for overlapping community detection based on edge encoding. Inf Sci 462:290-314. https://doi.org/10.1016/j. ins.2018.06.015

Bui K-HN, Cho S, Jung JJ, Kim J, Lee O-J, Na W (2019) A novel network virtualization based on data analytics in connected environment. J Ambient Intell Hum Comput. https://doi.org/10.1007/ s12652-018-1083-x (in this issue)

Carneiro D, Pinheiro AP, Novais P (2017) Context acquisition in auditory emotional recognition studies. J Ambient Intell Hum Comput 8(2):191-203. https://doi.org/10.1007/s12652-016-0391-2

Chouchani N, Abed M (2019) Enhance sentiment analysis on social networks with social influence analytics. J Ambient Intell Hum Comput. https://doi.org/10.1007/s12652-019-01234-0 (in this issue)

Derbas N, Dusserre E, Padro M, Segond F (2019) Eventfully safapp: hybrid approach to event detection for social media mining. J Ambient Intell Hum Comput. https://doi.org/10.1007/s12652-0181078-7 (in this issue)

Gonzalez-Pardo A, Jung JJ, Camacho D (2017) ACO-based clustering for Ego Network analysis. Future Gener Comput Syst 66:160-170. https://doi.org/10.1016/j.future.2016.06.033

Liao H, Xu Z, Herrera-Viedma E, Herrera F (2018) Hesitant fuzzy linguistic term set and its application in decision making: a stateof-the-art survey. Int J Fuzzy Syst 20(7):2084-2110. https://doi. org/10.1007/s40815-017-0432-9

Martín A, Rodríguez-Fernández V, Camacho D (2017) Evolving deep neural networks architectures for Android malware classification. In: Proceedings of evolutionary computation (CEC), 2017 IEEE congress, pp 1659-1666. https://doi.org/10.1109/ CEC.2017.7969501

Martín A, Rodríguez-Fernández V, Camacho D (2018) CANDYMAN: classifying Android malware families by modelling dynamic 
traces with Markov chains. Eng Appl Artif Intell 74:121-133. https://doi.org/10.1016/j.engappai.2018.06.006

Mezni H, Arab SA, Benslimane D, Benouaret K (2019) An evolutionary clustering approach based on temporal aspects for contextaware service recommendation. J Ambient Intell Hum Comput. https://doi.org/10.1007/s12652-018-1079-6 (in this issue)

Sani NS, Manthouri M, Farivar F (2019) A Multi-Objective Ant Colony Optimization Algorithm for Community Detection in Complex Networks. J Ambient Intell Hum Comput. https://doi. org/10.1007/s12652-018-1159-7 (in this issue)

Singh NK, Tomar DS, Sangaiah AK (2019) Sentiment analysis: a review and comparative analysis over social media. J Ambient Intell Hum Comput. https://doi.org/10.1007/s12652-018-0862-8 (in this issue)

Valdivia A, Cámara SM, Chaturvedi I, Luzón MV, Cambria E, Ong YS, Herrera F (2019) What do people think about this monument? Understanding negative reviews via deep learning, clustering and descriptive rules. J Ambient Intell Hum Comput. https://doi. org/10.1007/s12652-018-1150-3 (in this issue)

Villar P, Alemán I, Castillo L, Damas S, Cordón O (2017) A first approach to a fuzzy classification system for age estimation based on the pubic bone. In: Proceedings of fuzzy systems (FUZZIEEE), 2017 IEEE international conference, pp 1-6. https://doi. org/10.1109/FUZZ-IEEE.2017.8015760

Xylogiannopoulos K, Karampelas P, Alhajj R (2019) A password creation and validation system for social media platforms based on big data analytics. J Ambient Intell Hum Comput. https://doi. org/10.1007/s12652-019-01172-x (in this issue)

Publisher's Note Springer Nature remains neutral with regard to jurisdictional claims in published maps and institutional affiliations. 